\title{
Surgical treatment of nonalcoholic fatty liver disease in severely obese patients
}

This article was published in the following Dove Press journal:

Hepatic Medicine: Evidence and Research

29 October 2014

Number of times this article has been viewed

\section{Steven J Vander Naalt Juan P Gurria}

\section{AiXuan L Holterman}

University of Illinois College of Medicine at Peoria, Children's Hospital of Illinois, Department of Surgery/Pediatric Surgery, Peoria, IL, USA
Correspondence: AiXuan L Holterman University of Illinois College of Medicine at Peoria, Children's Hosptial of Illinois, 420 NE Glen Oak, Suite 20I,

Peoria, IL, USA 61603

Tel +l 3096243136

Fax +I 3096553948

Email aixuan.I.holterman@osfhealthcare. org

\begin{abstract}
Obesity is a multi-organ system disease with underlying metabolic abnormalities and chronic systemic inflammation. Nonalcoholic fatty liver disease (NAFLD) is a hepatic manifestation of obesity metabolic dysfunction and its associated cardiovascular- and liverrelated morbidities and mortality. Our current understanding of NAFLD pathogenesis, disease characteristics, the role of insulin resistance, chronic inflammation, gut-liver and gut-brain crosstalk and the effectiveness of pharmacotherapy is still evolving. Bariatric surgery significantly improves metabolic and NAFLD histology in severely obese patients, although its positive effects on fibrosis are not universal. Bariatric surgery benefits NAFLD through its metabolic effect on insulin resistance, inflammation, and insulinotropic and anorexinogenic gastrointestinal hormones. Further studies are needed to understand the natural course of NAFLD in severely obese patients and the role of weight loss surgery as a primary treatment for NAFLD.
\end{abstract}

Keywords: NAFLD, severe obesity, bariatric surgery

\section{Introduction}

Nonalcoholic fatty liver disease (NAFLD) occurs in both the young and old as well as in every ethnic population group. ${ }^{1}$ It is quickly becoming a world health problem as a function of the rise in obesity and as the relationship between NAFLD and obesity is recognized. ${ }^{2}$ NAFLD in obese patients is a frequent metabolic dysfunction with significant associated comorbidities. The incidence of NAFLD in the general population is variable, with a reported prevalence between $6 \%$ and $51 \%{ }^{3}$ In patients with obesity, NAFLD incidence is between $24 \%-98 \%{ }^{4}$ NAFLD patients have increased overall risk of death relative to the general population, ${ }^{5}$ with cardiovascular disease as the most common cause of death. ${ }^{6}$ The cardiac risks are predictable, considering the powerful association of NAFLD with metabolic syndrome (MS), and inflammation with atherosclerosis. ${ }^{5,7,8}$ NAFLD also has liver-related complications such as progression to cirrhosis and hepatocellular carcinoma. ${ }^{9}$ Once progression to nonalcoholic steatohepatitis (NASH) develops, there is a $4 \%-27 \%$ risk of cirrhosis over 10 years with a $12 \%$ risk of liver-related death. ${ }^{10}$ Currently, NAFLD is the number one cause of liver function abnormalities and chronic liver disease in adults. ${ }^{11}$ It is predicted to be the most common indication for liver transplantation over the next 10 years, making it an impending public health crisis of the century. Since obesity is a multi-organ system disease with chronic relapsing characteristics, NAFLD disease course is likely to follow the metabolic profile of obesity, necessitating a multidisciplinary approach combining lifestyle modifications with weight reduction, medications, and bariatric 
surgery. Early diagnosis and aggressive treatment of NAFLD is therefore indicated because of the associated morbidities. Severely obese patients are particularly at risk for liver-related mortality and should be the focus of treatment strategies for fatty liver disease. This article will provide a brief review of the underlying pathogenesis and current medical treatment as the background for an update on the current role of weight loss surgery on the outcome of NAFLD.

\section{Obesity and MS}

The epidemic of obesity has now become a global health issue. In the United States alone, the 2012 population data show that $32 \%$ of adults are overweight with $34.9 \%$ of adults considered to be obese. ${ }^{12}$ The prevalence did not differ between men and woman. The highest percentage of obesity was seen in the non-Hispanic black population with $47.8 \%$ being classified as obese. Current definitions for obesity are based on overall body mass index (BMI). The World Health Organization (WHO) defines obesity as BMI $\geq 30 \mathrm{~kg} / \mathrm{m}^{2}$. Obesity is further classified as Class I: BMI 30-34.9 kg/m², Class II: BMI 35-39.9 $\mathrm{kg} / \mathrm{m}^{2}$, and Class III: BMI $\geq 40 \mathrm{~kg} / \mathrm{m}^{2}$. Morbidly or severely obese patients have a BMI $\geq 40 \mathrm{~kg} / \mathrm{m}^{2}$. Currently, $6.6 \%$ of the US population is considered to be severely obese..$^{13}$ National Institutes of Health BMI criteria for weight loss surgery are BMI $>40 \mathrm{~kg} / \mathrm{m}^{2}$ or $\geq 35 \mathrm{~kg} / \mathrm{m}^{2}$ with one obesity-related comorbidity, such as fatty liver disease. Most of the complications of obesity are related to the associated MS. A review of MS can be found in many excellent in-depth articles. ${ }^{14,15}$ Briefly, MS is defined by the Adult Treatment Panel III ${ }^{14}$ or the International Diabetes Federation Task Force ${ }^{16}$ as the presence of three or more components of 1) severe obesity, central obesity by waist circumference, 2) type 2 diabetes mellitus, insulin resistance (IR) measured by fasting serum insulin and glucose, 3) hypertension, and 4) dyslipidemia. MS is a known metabolic predictor of atherosclerosis and cardiovascular mortality risks, ${ }^{17}$ placing obese patients at an increased hazard ratio for all causes of mortality. ${ }^{18} \mathrm{MS}$ has the central features of IR, visceral adiposity, dyslipidemia, endothelial dysfunction, hypercoagulability, chronic stress, and hypercortisolism. These global pathologic changes lead to an increased risk for diabetes mellitus, hypertension, dyslipidemia, ischemic heart disease, cerebrovascular disease, and liver disease. The underlying causes are not clear but excess visceral fat, adipocyte dysfunction, chronic low-grade inflammation, and genetic predisposition have been invoked. Because of the strong association between MS and NAFLD, NAFLD is considered to be part of the spectrum of obesityrelated metabolic dysfunctions. ${ }^{19}$

\section{NAFLD}

NAFLD is seen in $24 \%$ of the adult population. ${ }^{20}$ This figure rises to $74 \%$ in severely obese adult patients. ${ }^{21}$ NAFLD describes a spectrum of hepatic conditions ranging from simple steatosis (defined as hepatic fat infiltration in $>5 \%$ of the liver), to the inflammatory form of NAFLD or steatohepatitis (also called NASH) characterized by hepatic inflammation, necrosis, and hepatocyte ballooning, with or without fibrosis. NASH is associated with the risks for progression to end-stage cirrhosis and liver failure. ${ }^{22}$ The American Association for the Study of Liver Disease has proposed three classifications for NAFLD: 1) nonalcoholic fatty liver or simple steatosis without inflammation; 2) NASH, the advanced form of NAFLD, and 3) borderline NASH. ${ }^{22}$ When diagnosing NAFLD in obesity, other causes of fatty liver need to be excluded such as viral, infectious, drugs, toxins, autoimmune, and metabolic conditions (eg, cystic fibrosis, Wilson's disease). A careful social history must be taken to exclude excessive alcohol consumption $(>10 \mathrm{~g}$ of ethanol per day) as a cause of steatosis. ${ }^{23}$

Several histological grading systems for NAFLD based on liver biopsy have been developed, with the Brunt ${ }^{24}$ and the NASH-Clinical Research Network Activity Score (NAS) ${ }^{25}$ being the main cataloging systems. NAS classification relies on individual histologic scores of steatosis, inflammation, and hepatocyte ballooning. The more widely used Brunt grading and staging system classifies steatohepatitis into one of three categories: mild, moderate, or severe ${ }^{24}$ with a four-score staging system for fibrosis: perisinusoidal fibrosis (stage 1), periportal fibrosis (stage 2), bridging fibrosis (stage 3), and cirrhosis (stage 4).

Clinically, NAFLD is difficult to diagnose. The majority of patients are asymptomatic. However, some patients may complain of malaise, right upper quadrant abdominal pain, or hepatomegaly. Many of the clinical evaluations are undertaken in the absence of symptoms because of incidental abnormal liver function tests, especially alanine transaminase (ALT). ${ }^{26,27}$ Normal liver function tests, however, cannot be used exclusively to diagnose NAFLD, nor to rule out advanced NAFLD. ${ }^{28}$ Unfortunately, the progression of fatty liver disease is sometimes only apparent after the signs and symptoms of cirrhosis have already developed.

Noninvasive imaging techniques such as abdominal ultrasound, computed tomography, and magnetic resonance imaging can detect steatosis, although a negative test cannot rule out more advanced NAFLD. ${ }^{26,29}$

Diagnostic panels including FibroTest, NAFLD fibrosis score based on many variables such as age, BMI, 
hyperglycemia, platelet count, albumin, and aspartate aminotransferase/alanine transaminase ratio; and diagnostic biomarkers such as proinflammatory molecules, apoptosisassociated cytokeratin 18 fragments, and microRNA, ${ }^{30}$ have been advocated, but not universally accepted for a noninvasive identification of NAFLD patients with bridging fibrosis and or cirrhosis. ${ }^{31}$ Liver biopsy remains the gold standard for the diagnosis and the staging of NAFLD and NASH. ${ }^{1}$ Because of the cost, invasiveness, and morbidity of the procedure, it is not practical to perform a liver biopsy on all at-risk patients. Currently, there are no definitive guidelines to screen, image, or biopsy patients for NAFLD diagnosis. A liver biopsy should be considered in patients who are at an increased risk for having steatohepatitis and advanced fibrosis, ${ }^{22}$ such as individuals with obesity, MS, and persistently abnormal liver chemistries.

\section{Pathogenesis: obesity and NAFLD}

The pathogenesis of NAFLD has been aggressively researched but remains incompletely understood. IR and MS are most commonly associated with NAFLD. Because of the powerful association between MS and NAFLD, NAFLD is considered to be a manifestation of MS. Many recent articles have provided in-depth reviews of NAFLD molecular pathways. ${ }^{32-34}$ Research has centered on the relationship between three cell types: adipocytes, hepatocytes, and the intestinal epithelial cells. There are two proposed organ system interactions underlying NAFLD during obesity: the fat-liver interaction and the gut-liver interaction.

The fat-liver axis involves pathologic changes and adaptations of the hypertrophied fat-laden adipocytes during obesity and the development of IR. During obesity, adipocytes acquire a new proinflammatory molecular signature and abnormal free fatty acid release. ${ }^{35}$ Free fatty acids activate stress kinases such as c-Jun NH2 terminal kinase (JNK), which, in concert with proinflammatory cytokines, cause derangement in the insulin-signaling pathway in adipocytes and hepatocytes. ${ }^{36-38}$ These pathways impair pancreatic endocrine cell function to further exacerbate IR. Both the stress kinases and hyperinsulinemia additionally cause abnormal responses of hepatic lipogenic transcription factors, leading to excess gluconeogenesis, de novo lipid synthesis, and abnormal lipid transport. ${ }^{39,40}$ Lipid burden in hepatocytes induces cellular production of oxygen-free radicals. Oxidative stress activates JNK, perpetuates IR, induces mitochondrial and endoplasmic reticulum stress, cell death, and injures hepatocytes. ${ }^{41}$ Inflammation and phagocytosis of apoptotic hepatocytes by macrophage results in the activation of hepatic stellate cells into myofibroblast-like cells to initiate a profibrogenic response. ${ }^{42}$

Pathologies underlying obesity and NAFLD may be more complicated than simple abnormal fat-liver interactions. The second proposed mechanism of NAFLD development in obesity relates to the gut-liver crosstalk. The gut is a large lymphoid organ with an active, diverse bacterial microflora. This microflora supports intestinal metabolic, digestive, hormonal, and trophic activities. It also interacts with the host immune response to pathogens. With obesity, increased consumption of fructose and lipids also alters the microbiota of the gut. It has been shown that patients with NAFLD have increased intestinal permeability and small intestinal bacterial overgrowth. ${ }^{43}$ As the first organ responder to bacterial translocation, the liver innate immune response and hepatic inflammation are induced, ${ }^{44}$ furthering hepatic IR.

Additional intestinal molecular pathways are regulated by bile acids and gut peptides such as incretins (glucagon-like peptide-1 [GLP-1] and glucose-dependent insulinotropic polypeptide). Incretins ${ }^{45}$ are nutrient sensors with diverse effects on cardiovascular function, satiety, gastric emptying, insulin production, and glucagon release to affect hepatic IR and lipid metabolism. ${ }^{46,47}$ As major ligands for G-proteincoupled receptor Transforming Growth Factor 5, bile acids indirectly activate GLP-1 to improve insulin sensitivity. Bile acids also bind to lipid-sensing nuclear hormone receptors such as farnesoid $\mathrm{X}$ receptor to modulate hepatic cholesterol, lipoprotein, and glucose metabolism. ${ }^{48}$

More recently, non-coding RNA molecules known as microRNA involved in the regulation of gene transcription have been linked to the pathogenesis, progression, and severity of NAFLD through their effects in cell growth, apoptosis, and inflammation as well as lipid and fatty acid metabolism, suggesting that they can be used as biomarkers or as targets for therapy. ${ }^{49}$ Consistent with this, visceral fat from morbidly obese patients has been shown to have a signature profile of microRNA which correlates with the severity of NAFLD. ${ }^{50,51}$

\section{Management of NAFLD Pharmacologic}

Nonoperative management of fatty liver disease has centered on the treatment of obesity and of the individual components of MS. Encouraging results have been obtained with lifestyle modifications in obese patients with fatty liver disease and the mainstay of treatment for steatosis remains weight reduction. Eckard et al have shown that diets low in fat or processed carbohydrates and moderate exercise can be effective in 
improving steatosis in NAFLD as shown by follow-up liver biopsy. ${ }^{52}$ Current recommendations call for a loss of 5\% of excess weight for steatosis and a 10\% excess weight loss for $\mathrm{NASH},{ }^{53}$ but long-term compliance is problematic and the effectiveness of weight loss for NASH is inconclusive. ${ }^{54-56}$ Lifestyle modifications have not been shown to be a reliable long-term management strategy in the treatment of obesity for the majority of individuals. ${ }^{57}$

Since steatosis has been considered to be a benign, non-progressive disease, pharmacologic or invasive interventions are not indicated. The treatment of NASH has focused on a variety of modalities including lifestyle modification, and pharmacologic and surgical interventions. No current cure for NASH exists, but many studies have investigated pharmacotherapy for the treatment of the IR and hyperlipidemia components of MS to alter the course of NASH. Several pharmacologic treatments such as orlistat, ${ }^{58}$ pioglitazone, ${ }^{59}$ metformin, ${ }^{60}$ atorvastatin, ${ }^{61}$ ursodiol, ${ }^{61}$ vitamin E, ${ }^{62}$ omega-3 fatty acids, ${ }^{63}$ and many others have been proposed. Improvements in liver biopsy findings have been demonstrated with pioglitazone, rosiglitazone, atorvastatin, and ursodiol. However, these therapies have associated pharmacologic adverse effects and the efficacies have not been consistently reproduced in clinical trials, ${ }^{64,65}$ putting in question their role as definitive treatment for NASH. Integrative analyses of obesity-associated molecular pathways are being actively pursued toward identification of genetic, transcriptomic, and proteonomic signatures to provide drug-based interventions. ${ }^{66}$ With insufficient general acceptance for NASH pharmacologic treatment, weight reduction through bariatric surgery is increasingly used as a therapeutic modality to improve the metabolic dysfunction with secondary gain on improving NASH. It is worth noting that the lipostatic model of body mass regulation postulates that each individual has a central set-point for hunger and energy balance. ${ }^{67}$ This may account for weight gain recidivism after the initial successful weight loss in some bariatric patients with long-term follow-up. ${ }^{68}$ It follows that some obese patients may benefit from multipronged weight loss approaches incorporating bariatric procedures with maintenance pharmacotherapeutics such as GLP-1 analogs to sustain long-term weight loss and metabolic benefits.

\section{Bariatric surgery}

Bariatric surgery is currently indicated for severely obese individuals with a $\mathrm{BMI}>40 \mathrm{~kg} / \mathrm{m}^{2}$ or with a $\mathrm{BMI} \geq 35$ $\mathrm{kg} / \mathrm{m}^{2}$ and obesity-related comorbid conditions. Weight loss by bariatric surgery is effective in improving and/or resolving metabolic abnormalities with up to a $40 \%$ reduction in long-term obesity-related morbidity. ${ }^{69}$ With a causal link established between NAFLD and the obesity-related MS, bariatric surgery interventions promoting weight loss would be expected to improve NAFLD. No randomized controlled studies have been performed to specifically examine the efficacy of bariatric surgery as a primary indication for the treatment of NAFLD, but several studies have been published addressing NAFLD as a secondary outcome of bariatric surgery. A meta-analysis of 21 studies examining the effect of surgery on NAFLD in morbidly obese patients reported significant improvement or resolution of steatosis (92\%), NASH histology ( $82 \%)$, and fibrosis $(66 \%) .{ }^{70}$ Because progression of fibrosis has been noted in some patients, the article concluded that bariatric surgery impact on NASH disease course remains to be proven. A recent review of the bariatric literature provided similar findings of consistent histological improvement in steatosis, inflammation, and fibrosis following weight loss, but with new onset or progression of NASH or of hepatic fibrosis in some patients. ${ }^{71}$ The American College of Gastroenterology, the American Gastroenterological Association, and the American Association for the Study of Liver Diseases published the consensus statement that while bariatric surgery is not contraindicated in otherwise eligible obese patients with NAFLD, it is not an "established option for NASH treatment." 22 Concerns about progression of NAFLD to cirrhosis described in patients undergoing jejunoileal bypass ${ }^{72,73}$ or with rapid weight loss following bariatric surgery ${ }^{74,75}$ provide the additional rationale for the position that the safety and efficacy of bariatric surgery in patients with established cirrhosis are not proven. It is worth mentioning that a prospective study of 1,775 bariatric patients with a matched medical cohort reported sustained improvement in liver function enzymes following bariatric surgery at 2 and 10 years of follow-up. ${ }^{76}$ In the absence of long-term follow-up for liver-related comorbidities, the current disease course assessment for NAFLD has been limited to short term follow up of liver histology evaluation as a primary outcome. We conducted a review of the published bariatric reports using liver biopsy as the gold standard to diagnose and evaluate NAFLD. Most of the publications are retrospective analyses with many confounding biases, small sample size, lack of sequential systematic follow-up biopsies, variable methodologies for reporting histological features, and scant information on patient selection criteria and associated metabolic data. With these limitations in mind, the effect of the different bariatric surgical techniques: 1) 
restrictive or 2) restrictive and malabsorptive procedures on NAFLD histology are summarized below with highlights on articles of particular interest.

\section{Restrictive}

The restrictive procedures reduce the stomach volume and thereby, caloric intake. A 2009 prospective study by Mathurin et al described 381 consecutive patients with initial liver biopsy at the time of bariatric surgery, of which $56 \%$ underwent gastric banding. ${ }^{77}$ The majority of the patients underwent planned follow-up liver biopsies at 1-year (85\% patients) and 5-year (81\% patients). ${ }^{77}$ Substantial weight loss and improvement in metabolic parameters were achieved. The global NAS scores, the individual steatosis and ballooning degeneration scores and the incidence of NASH all significantly improved. The inflammation and fibrosis NAS scores were unchanged, although $96 \%$ of the patients had low fibrosis scores of $\leq 1$ (no fibrosis or focal fibrosis), and fibrosis improved in $80 \%$ of the patients. At 1 and 5 years, 4/258 patients and 2/203 patients with underlying fibrosis progressed to bridging fibrosis. No patient developed de novo bridging fibrosis except for a patient with alcoholic abuse. Patients with persistent IR were more likely to have less favorable evolution of their hepatic disease.

A retrospective analysis by Luyckx et al reviewed $69 / 528$ vertical gastroplasty patients who had paired liver biopsies within an average of 3 years of the initial gastroplasty biopsies. ${ }^{21}$ Steatosis significantly improved. Perisinusoidal inflammation worsened in $26 \%$ with $88 \%$ characterized as mild and $13 \%$ as moderate disease. Stratopoulous et al's retrospective evaluation of 51/216 gastroplasty patients with baseline and subsequent liver biopsies at an average of 18 months $^{78}$ showed regression of NASH, and marked improvement in steatosis, inflammation, and fibrosis; with unchanged fibrosis in $41 \%$ but worse fibrosis in $12 \%$ (six patients). Dixon et al reported 60 patients with follow-up biopsies showing consistent lower histologic NAS scores in steatosis, inflammation, and fibrosis with $80 \%$ resolution of NASH and $13 \%$ stable disease in the 30 patients with baseline NASH. ${ }^{79}$ No liver disease progression was noted.

\section{Malabsorptive/restrictive}

Two malabsorptive/restrictive procedures, the biliopancreatic diversion with duodenal switch (BPD-DS) as well as the Roux-en-Y gastric bypass (RYGB) capitalize on caloric intake reduction from the restrictive component of the procedure and nutrient malabsorption by diverting the flow of intestinal enzymes and ingested nutrition to enhance weight loss. Both surgical approaches have become widely accepted in the treatment of morbid obesity because they provide rapid and substantial weight reduction.

Significant responses in MS and its related pathologies have been demonstrated with the BPD-DS. ${ }^{80}$ For example, diabetes is cured in over $90 \%$ of patients based on medication cessation. Reports of the effect of BPD-DS on NAFLD are limited. The available data come from two large studies by Kral et al in 2004 and Keshishian et al in 2005..$^{81,82}$ Kral et al prospectively studied 104/689 BPD-DS patients with follow-up liver biopsy at $41 \pm 25$ months. Steatosis and fibrosis semiquantitative grading decreased with weight loss. Fibrosis increased in $42 \%$ of the patients, but mostly from grade 0 to 1 . Three patients had new onset cirrhosis but they also had underlying biliary tract obstruction, alcohol abuse, and intractable diarrhea. Notably, cirrhosis was encountered in 11 or $2 \%$ of the patients at the time of BPD-DS. At an average of 112 months of follow-up, the remarkable finding was that improvement or regression of cirrhosis occurred in $9 / 11$ patients. The remaining two patients continued to have disease progression, one with intractable diarrhea and pancreatitis and one with hemosiderosis. These data suggest that severely obese patients with compensated cirrhosis may benefit from weight loss procedures. Keshishian et al retrospectively evaluated 78/697 patients with follow-up liver biopsy between 6 and 36 postoperative months after BPDDS. ${ }^{82}$ NASH histology initially worsened with concurrent rise in liver function tests at 6 months but steadily improved at follow-up. The initial histological deterioration was attributed to the initial acute weight loss.

The majority of the literature assessing the effect of bariatric surgery on the course of NAFLD involves RYGB patients because it is the most commonly performed malabsorptive/restrictive procedure. Thirteen studies investigated the changes in liver histology after RYGB. ${ }^{83-94}$ Four studies by Furuya et al, ${ }^{90}$ Barker et al, ${ }^{87}$ Tai et al,,${ }^{95}$ and de Almeida et $\mathrm{al}^{89}$ are worth mentioning because of the availability of planned paired biopsies. In Furuya et al's prospective study, 18 patients underwent repeat control percutaneous liver biopsies at 2 years post-surgery. Significant improvement of the metabolic parameters ${ }^{90}$ paralleled lower overall NAS and fibrosis scores for all patients, with 75\% regression of NASH and 50\% regression of fibrosis, and no progression of disease in all patients. Similarly, Tai et al prospectively evaluated 21 patients with planned repeat liver biopsies at 1 year post-RYGB. Improvement in all histological categories was seen with no disease progression. Barker et al performed follow-up biopsies in 19 patients 
with prior diagnosis of NASH after an average interval of 21 months. NASH resolved in $89 \%$ of the patients. One patient had mild worsening of fibrosis score from 0 to 1 with an additional patient having portal fibrosis score progression from stage 2 to stage $3 .{ }^{87}$ In de Almeida's study, 16 patients with repeat liver biopsies at a median follow-up of 20 months after having been diagnosed with NASH from the initial biopsy had similar decreases in NAS scores in all categories. NASH regressed in 15/16 of the patients and improved in $1 / 16{ }^{89}$

Publications reporting post-surgical progression of hepatic fibrosis are worth mentioning. They were all retrospective RYGB studies where repeat biopsies were performed on a very small subset of prior bariatric patients because of the opportunities for biopsies at the time of reoperative surgery. A common theme is the overall improvement of metabolic results, steatosis, NASH parameters, and the lack of change in portal tract pathologies except for isolated cases of new onset or progression of liver fibrosis. In Mattar et al's study of 70/3,000 bariatric patients with repeat biopsies, the percentage of patients with grade $1 \mathrm{NASH}$ increased from $26 \%$ to $50 \%$ on repeat biopsy at an average of 15 postoperative months. ${ }^{85}$ In 91/613 of Silverman et al's liver rebiopsies at a postoperative average of 18 months, one patient had new perisinusoidal fibrosis, while fibrosis improved or regressed in the remaining patients. ${ }^{83}$ Of the $16 / 557$ RYGB patients with rebiopsies at 18 months in Csendes et al's report, one patient had progression from steatosis to pericellular fibrosis. ${ }^{88}$ In Moretto et al's study of 78/644 RYGB patients, ${ }^{93}$ baseline NASH histology regressed in 23/39 patients and fibrosis improved in 52\% of patients at an average of 12 months after the first biopsy. Of note, 2/79 patients developed new onset fibrosis stage 1 and 2 , and three patients progressed from stage 1 to stage 3 bridging fibrosis. Liu et al's report of 39 patients with repeat liver biopsies showed that NAS grading improved for all histological categories with $100 \%$ resolution of NASH. ${ }^{91}$ Two patients had increased lobular inflammation from grade 1 to 2 , and six patients developed new portal inflammation. In all of these studies, the basis for disease progression in the individual patients was not apparent.

In summary, while rigorous data are limited, the vast majority of bariatric patients have consistent improvement of steatosis, inflammation, and fibrosis and no disease progression, providing strong support for bariatric salutary effect on obesity-related NASH. For the majority of the few patients in whom inflammation or fibrosis worsened after restrictive bariatric surgery, the degree of histological changes was mild. Patient-specific factors such as rapid weight loss and loss of macronutrients, bacterial overgrowth, poor metabolic response to weight reduction, discordant results between the first and the repeat biopsies from sampling variability and interpretation error inherent to liver biopsies, ${ }^{96}$ histological progression as the natural course of the fatty liver disease, procedure-related specific complications are all possible causes for disease progression that cannot be substantiated from the articles. Severely obese patients with NASH therefore should not be excluded from undergoing restrictive bariatric surgery to treat the underlying obesity and metabolic dysfunction because of the secondary gain in liver histologic improvement. Since simple steatosis is considered to be a benign histological finding, it does not meet the criterion as an indication for National Institutes of Health bariatric guidelines in patients with $\mathrm{BMI} \geq 35 \mathrm{~kg} / \mathrm{m}^{2}$.

Due to BPD-DS's higher perioperative and postoperative morbidity, its underlying procedure-related risks for bacterial stasis from blind loop syndrome, (which may further exacerbate liver disease), and its potential association with an acute decline in NAFLD histology from drastic weight loss, this procedure should be used with caution in patients at risk for and/or in patients with advanced NAFLD. Quality prospective studies should be performed to definitively address the safety of BPD-DS in NAFLD patients.

\section{Potential mechanisms of bariatric surgery effect on NAFLD}

The beneficial effects of bariatric surgery on metabolic dysfunction have redefined weight loss bariatric surgery as "metabolic surgery," differences in the degree of metabolic efficacy. ${ }^{98}$ Its mechanisms are more complex than simple weight reduction from caloric restriction and malabsorption. Following bariatric surgery, the loss of excess body fat can enhance adipocyte function, improve IR and systemic inflammation to restore metabolic balance; and the alteration in the intestinal microenvironment can modify the course of NAFLD. The intestinal bypass procedures may have additional hormonal advantages such as alterations in gastrointestinal peptides with the net effect of promoting satiety and insulin sensitivity.

A 2004 meta-analysis study demonstrated an overall $61.2 \%$ reduction in excess body fat following restrictive or restrictive/malabsorptive procedures. ${ }^{99}$ Since excess body fat has been linked to IR and MS, lower fat mass following 
weight reduction in bariatric patients decreases lipid burden (as demonstrated by declines in circulating lipid profiles including fatty acids, low-density lipoprotein, triglycerides, and lipoprotein A levels) ${ }^{100}$ and improves IR. ${ }^{101}$ Similarly, systemic inflammation is attenuated as shown by favorable alteration in the profile of circulating proinflammatory and anti-inflammatory cytokines such as C-reactive protein, interleukin 6 , interleukin 18 , and tumor necrosis factor $\alpha,{ }^{102,103}$ or anti-atherogenic, anti-inflammatory, and anti-diabetogenic adiponectin ${ }^{104}$ following gastric bypass.

In the intestines, changes in the microbiota ${ }^{105}$ (such as increased Proteobacteria and reduced Firmicutes species) ${ }^{106}$ favor a non-obesogenic profile, improve the energy profile, and heighten insulin sensitivity after bariatric surgery. Likewise, secondary increases in the levels of gut incretins GLP-1, glucose-dependent insulinotropic polypeptide, polypeptide YY, and oxyntomodulin in response to proximal intestinal exclusion and accelerated nutrient delivery to the distal intestines following RYGB can improve hepatic IR. ${ }^{107,108}$ In the liver, GLP-1 promotes hepatic lipid export and oxidation, reduces hepatic proinflammatory cytokines, and decreases endoplasmic reticulum stress. ${ }^{109}$ Along with anorexigenic ghrelin ${ }^{110}$ and polypeptide YY, ${ }^{111}$ GLP-1 has additional extraintestinal effects on the gut-brain axis ${ }^{112}$ to regulate central satiety, and diminish hunger and food intake. The combinatorial effects of bariatric surgery in reducing fat mass, altering neurohormonal regulation of appetite, or favoring less obesogenic intestinal microbiota may help enhance insulin sensitivity and diminish systemic inflammation to improve NAFLD. Further studies are required to prove the underlying mechanisms.

\section{Conclusion}

Morbid obesity has become a worldwide epidemic, bringing with it a multitude of metabolic abnormalities including NAFLD. Pharmacologic interventions for NAFLD targeting inflammation or IR remain unproven. Because of safety concerns, lack of consistent efficacy, and the requirement that patients are enrolled in clinical trials for drug eligibility, pharmacotherapy has limited practical clinical use. The role of bariatric surgery as a primary indication for NAFLD treatment has yet to be systematically studied. However, despite imperfect information, the preponderance of the data suggests that NASH improves following bariatric surgery, with an outcome closely linked to the metabolic end results. The benefits of bariatric surgery on the course of NASH are not universally consistent, calling for the need for quality longitudinal studies to better understand the natural history of NAFLD after bariatric surgery. Without long-term follow-up, the sustainability of these outcomes also remains unclear. Further information is needed on the safety and efficacy of specific procedurerelated bariatric surgery in improving NASH histology in severely obese patients, with particular attention to the subset of patients who continues to have NAFLD disease progression. Additional studies are also needed to facilitate further understanding of the pathogenesis of NAFLD and the mechanisms of bariatric surgery effects on NAFLD. In the absence of effective noninvasive alternatives, bariatric surgery offered within the context of well-designed studies offers the most promise in the treatment of NAFLD in severely obese patients.

\section{Disclosure}

The authors report no conflicts of interest in this work.

\section{References}

1. Adams LA, Angulo P. Treatment of non-alcoholic fatty liver disease. Postgrad Med J. 2006;82(967):315-322.

2. Schwenger KJ, Allard JP. Clinical approaches to non-alcoholic fatty liver disease. World J Gastroenterol. 2014;20(7):1712-1723.

3. Vernon G, Baranova A, Younossi ZM. Systematic review: the epidemiology and natural history of non-alcoholic fatty liver disease and non-alcoholic steatohepatitis in adults. Aliment Pharmacol Ther. 2011;34(3):274-285.

4. Gholam PM, Flancbaum L, Machan JT, Charney DA, Kotler DP. Nonalcoholic fatty liver disease in severely obese subjects. Am J Gastroenterol. 2007;102(2):399-408.

5. Söderberg C, Stål P, Askling J, et al. Decreased survival of subjects with elevated liver function tests during a 28-year follow-up. Hepatology. 2010;51(2):595-602.

6. Ekstedt M, Franzén LE, Mathiesen UL, et al. Long-term follow-up of patients with NAFLD and elevated liver enzymes. Hepatology. 2006;44(4):865-873.

7. Eckel RH, Alberti KG, Grundy SM, Zimmet PZ. The metabolic syndrome. Lancet. 2010;375(9710):181-183.

8. Tarantino G, Caputi A. JNKs, insulin resistance and inflammation: A possible link between NAFLD and coronary artery disease. World $J$ Gastroenterol. 2011;17(33):3785-3794.

9. Adams LA, Lymp JF, St Sauver J, et al. The natural history of nonalcoholic fatty liver disease: a population-based cohort study. Gastroenterology. 2005;129(1):113-121.

10. Starley BQ, Calcagno CJ, Harrison SA. Nonalcoholic fatty liver disease and hepatocellular carcinoma: a weighty connection. Hepatology. 2010;51(5):1820-1832.

11. Angulo P. GI epidemiology: nonalcoholic fatty liver disease. Aliment Pharmacol Ther. 2007;25(8):883-889.

12. Ogden CL, Carroll MD, Kit BK, Flegal KM. Prevalence of obesity among adults: United States, 2011-2012. NCHS Data Brief. 2013;(131):1-8.

13. Sturm R, Hattori A. Morbid obesity rates continue to rise rapidly in the United States. Int J Obes (Lond). 2013;37(6):889-891.

14. Grundy SM, Cleeman JI, Daniels SR, et al. Diagnosis and management of the metabolic syndrome: an American Heart Association/National Heart, Lung, and Blood Institute scientific statement: Executive Summary. Crit Pathw Cardiol. 2005;4(4):198-203.

15. Kaur J. A comprehensive review on metabolic syndrome. Cardiol Res Pract. 2014;2014:943162. 
16. Alberti KG, Eckel RH, Grundy SM, et al; International Diabetes Federation Task Force on Epidemiology and Prevention; Hational Heart, Lung, and Blood Institute; American Heart Association; World Heart Federation; International Atherosclerosis Society; International Association for the Study of Obesity. Harmonizing the metabolic syndrome: a joint interim statement of the International Diabetes Federation Task Force on Epidemiology and Prevention; National Heart, Lung, and Blood Institute; American Heart Association; World Heart Federation; International Atherosclerosis Society; and International Association for the Study of Obesity. Circulation. 2009;120(16): $1640-1645$.

17. Treeprasertsuk S, Lopez-Jimenez F, Lindor KD. Nonalcoholic fatty liver disease and the coronary artery disease. Dig Dis Sci. 2011;56(1):35-45.

18. Berrington de Gonzalez A, Hartge P, Cerhan JR, et al. Body-mass index and mortality among 1.46 million white adults. $N$ Engl $J$ Med. 2010;363(23):2211-2219.

19. Tarantino G, Finelli C. What about non-alcoholic fatty liver disease as a new criterion to define metabolic syndrome? World J Gastroenterol. 2013;19(22):3375-3384.

20. Angulo P. Treatment of nonalcoholic fatty liver disease. Ann Hepatol. 2002;1(1):12-19.

21. Luyckx FH, Desaive C, Thiry A, et al. Liver abnormalities in severely obese subjects: effect of drastic weight loss after gastroplasty. Int $J$ Obes Relat Metab Disord. 1998;22(3):222-226.

22. Chalasani N, Younossi Z, Lavine JE, et al; American Gastroenterological Association; American Association for the Study of Liver Diseases; American College of Gastroenterologyh. The diagnosis and management of non-alcoholic fatty liver disease: practice guideline by the American Gastroenterological Association, American Association for the Study of Liver Diseases, and American College of Gastroenterology. Gastroenterology. 2012;142(7):1592-1609.

23. Ahmed M, Byrne C. Non-alcoholic fatty liver disease. In: Byrne CD, Wild SH, editors. The Metabolic Syndrome. 2nd ed. Oxford, UK: WileyBlackwell; 2011:245-277.

24. Brunt EM, Janney CG, Di Bisceglie AM, Neuschwander-Tetri BA, Bacon BR. Nonalcoholic steatohepatitis: a proposal for grading and staging the histological lesions. Am J Gastroenterol. 1999;94(9): 2467-2474.

25. Kleiner DE, Brunt EM, Van Natta M, et al; Nonalcoholic Steatohepatitis Clinical Research Network. Design and validation of a histological scoring system for nonalcoholic fatty liver disease. Hepatology. 2005;41(6): 1313-1321.

26. Sass DA, Chang P, Chopra KB. Nonalcoholic fatty liver disease: a clinical review. Dig Dis Sci. 2005;50(1):171-180.

27. Sorbi D, Boynton J, Lindor KD. The ratio of aspartate aminotransferase to alanine aminotransferase: potential value in differentiating nonalcoholic steatohepatitis from alcoholic liver disease. Am J Gastroenterol. 1999;94(4):1018-1022.

28. Browning JD, Szczepaniak LS, Dobbins R, et al. Prevalence of hepatic steatosis in an urban population in the United States: impact of ethnicity. Hepatology. 2004;40(6):1387-1395.

29. Saadeh S, Younossi ZM, Remer EM, et al. The utility of radiological imaging in nonalcoholic fatty liver disease. Gastroenterology. 2002;123(3):745-750.

30. Pearce SG, Thosani NC, Pan JJ. Noninvasive biomarkers for the diagnosis of steatohepatitis and advanced fibrosis in NAFLD. Biomarker Res. 2013;1(1):7.

31. Musso G, Gambino R, Cassader M, Pagano G. Meta-analysis: natural history of non-alcoholic fatty liver disease (NAFLD) and diagnostic accuracy of non-invasive tests for liver disease severity. Ann Med. 2011;43(8):617-649.

32. Attar BM, Van Thiel DH. Current concepts and management approaches in nonalcoholic fatty liver disease. ScientificWorldJournal. 2013;2013:481893.

33. Schuppan D, Schattenberg JM. Non-alcoholic steatohepatitis: pathogenesis and novel therapeutic approaches. $J$ Gastroenterol Hepatol. 2013;28 Suppl 1:68-76.
34. Wree A, Broderick L, Canbay A, Hoffman HM, Feldstein AE. From NAFLD to NASH to cirrhosis-new insights into disease mechanisms. Nat Rev Gastroenterol Hepatol. 2013;10(11):627-636.

35. Edmison J, McCullough AJ. Pathogenesis of non-alcoholic steatohepatitis: human data. Clin Liver Dis. 2007;11(1):75-104, ix.

36. Wajant $\mathrm{H}$, Pfizenmaier K, Scheurich P. Tumor necrosis factor signaling. Cell Death Differ. 2003;10(1):45-65.

37. Dresner A, Laurent D, Marcucci M, et al. Effects of free fatty acids on glucose transport and IRS-1-associated phosphatidylinositol 3-kinase activity. J Clin Invest. 1999;103(2):253-259.

38. Guerre-Millo M. Adipose tissue and adipokines: for better or worse. Diabetes Metab. 2004;30(1):13-19.

39. Horton JD, Shimomura I, Ikemoto S, Bashmakov Y, Hammer RE. Overexpression of sterol regulatory element-binding protein-1a in mouse adipose tissue produces adipocyte hypertrophy, increased fatty acid secretion, and fatty liver. J Biol Chem. 2003;278(38): 36652-36660.

40. Shimomura I, Hammer RE, Richardson JA, et al. Insulin resistance and diabetes mellitus in transgenic mice expressing nuclear SREBP-1c in adipose tissue: model for congenital generalized lipodystrophy. Genes Dev. 1998;12(20):3182-3194.

41. Ozcan U, Cao Q, Yilmaz E, et al. Endoplasmic reticulum stress links obesity, insulin action, and type 2 diabetes. Science. 2004;306(5695): 457-461.

42. Farrell GC, van Rooyen D, Gan L, Chitturi S. NASH is an Inflammatory Disorder: Pathogenic, Prognostic and Therapeutic Implications. Gut Liver. 2012;6(2):149-171.

43. Miele L, Valenza V, La Torre G, et al. Increased intestinal permeability and tight junction alterations in nonalcoholic fatty liver disease. Hepatology. 2009;49(6):1877-1887.

44. Alisi A, Manco M, Devito R, Piemonte F, Nobili V. Endotoxin and plasminogen activator inhibitor-1 serum levels associated with nonalcoholic steatohepatitis in children. J Pediatr Gastroenterol Nutr. 2010;50(6):645-649.

45. Mells JE, Anania FA. The role of gastrointestinal hormones in hepatic lipid metabolism. Semin Liver Dis. 2013;33(4):343-357.

46. Zarrinpar A, Loomba R. Review article: the emerging interplay among the gastrointestinal tract, bile acids and incretins in the pathogenesis of diabetes and non-alcoholic fatty liver disease. Aliment Pharmacol Ther. 2012;36(10):909-921.

47. Lee J, Hong SW, Rhee EJ, Lee WY. GLP-1 Receptor Agonist and Non-Alcoholic Fatty Liver Disease. Diabetes Metab J. 2012;36(4):262-267.

48. Fuchs M. Non-alcoholic fatty liver disease: the bile acid-activated farnesoid $\mathrm{X}$ receptor as an emerging treatment target. $J$ Lipids. 2012;2012:934396.

49. Ferreira DM, Simão AL, Rodrigues CM, Castro RE. Revisiting the metabolic syndrome and paving the way for microRNAs in non-alcoholic fatty liver disease. FEBS J. 2014;281(11):2503-2524.

50. Castro RE, Ferreira DM, Afonso MB, et al. miR-34a/SIRT1/p53 is suppressed by ursodeoxycholic acid in the rat liver and activated by disease severity in human non-alcoholic fatty liver disease. $J$ Hepatol. 2013;58(1):119-125.

51. Sharma H, Estep M, Birerdinc A, et al. Expression of genes for microRNA-processing enzymes is altered in advanced non-alcoholic fatty liver disease. J Gastroenterol Hepatol. 2013;28(8):1410-1415.

52. Eckard C, Cole R, Lockwood J, et al. Prospective histopathologic evaluation of lifestyle modification in nonalcoholic fatty liver disease: a randomized trial. Therap Adv Gastroenterol. 2013;6(4):249-259.

53. Masuoka HC, Chalasani N. Nonalcoholic fatty liver disease: an emerging threat to obese and diabetic individuals. Ann NY Acad Sci. 2013;1281:106-122.

54. Palmer M, Schaffner F. Effect of weight reduction on hepatic abnormalities in overweight patients. Gastroenterology. 1990;99(5):1408-1413.

55. Harrison SA, Fecht W, Brunt EM, Neuschwander-Tetri BA. Orlistat for overweight subjects with nonalcoholic steatohepatitis: A randomized, prospective trial. Hepatology. 2009;49(1):80-86. 
56. Wong VW, Chan RS, Wong GL, et al. Community-based lifestyle modification programme for non-alcoholic fatty liver disease: a randomized controlled trial. J Hepatol. 2013;59(3):536-542.

57. Wing RR, Phelan S. Long-term weight loss maintenance. Am J Clin Nutr. 2005;82(Suppl 1):222S-225S

58. Harrison SA, Fincke C, Helinski D, Torgerson S, Hayashi P. A pilot study of orlistat treatment in obese, non-alcoholic steatohepatitis patients. Aliment Pharmacol Ther. 2004;20(6):623-628.

59. Belfort R, Harrison SA, Brown K, et al. A placebo-controlled trial of pioglitazone in subjects with nonalcoholic steatohepatitis. $N$ Engl J Med. 2006;355(22):2297-2307.

60. Uygun A, Kadayifci A, Isik AT, et al. Metformin in the treatment of patients with non-alcoholic steatohepatitis. Aliment Pharmacol Ther. 2004;19(5):537-544.

61. Kiyici M, Gulten M, Gurel S, et al. Ursodeoxycholic acid and atorvastatin in the treatment of nonalcoholic steatohepatitis. Can J Gastroenterol. 2003;17(12):713-718.

62. Bugianesi E, Gentilcore E, Manini R, et al. A randomized controlled trial of metformin versus vitamin $\mathrm{E}$ or prescriptive diet in nonalcoholic fatty liver disease. Am J Gastroenterol. 2005;100(5):1082-1090.

63. Janczyk W, Socha P, Lebensztejn D, et al. Omega-3 fatty acids for treatment of non-alcoholic fatty liver disease: design and rationale of randomized controlled trial. BMC Pediatr. 2013;13:85.

64. Sanyal AJ, Mofrad PS, Contos MJ, et al. A pilot study of vitamin E versus vitamin $\mathrm{E}$ and pioglitazone for the treatment of nonalcoholic steatohepatitis. Clin Gastroenterol Hepatol. 2004;2(12):1107-1115.

65. Lindor KD, Kowdley KV, Heathcote EJ, et al. Ursodeoxycholic acid for treatment of nonalcoholic steatohepatitis: results of a randomized trial. Hepatology. 2004;39(3):770-778.

66. Freudenberg JM, Rajpal N, Way JM, Magid-Slav M, Rajpal DK Gastrointestinal weight-loss surgery: glimpses at the molecular level. Drug Discov Today. 2013;18(13-14):625-636.

67. Speakman JR, Levitsky DA, Allison DB, et al. Set points, settling points and some alternative models: theoretical options to understand how genes and environments combine to regulate body adiposity. Dis Model Mech. 2011;4(6):733-745.

68. Grattan BJ Jr, Connolly-Schoonen J. Addressing weight loss recidivism: a clinical focus on metabolic rate and the psychological aspects of obesity. ISRN Obes. 2012;2012:567530.

69. Sjöström L, Lindroos AK, Peltonen M, et al; Swedish Obese Subjects Study Scientific Group. Lifestyle, diabetes, and cardiovascular risk factors 10 years after bariatric surgery. $N$ Engl J Med. 2004;351(26): 2683-2693.

70. Mummadi RR, Kasturi KS, Chennareddygari S, Sood GK. Effect of bariatric surgery on nonalcoholic fatty liver disease: systematic review and meta-analysis. Clin Gastroenterol Hepatol. 2008;6(12): 1396-1402.

71. Hafeez S, Ahmed MH. Bariatric surgery as potential treatment for nonalcoholic fatty liver disease: a future treatment by choice or by chance? J Obes. 2013;2013:839275.

72. Peters RL, Gay T, Reynolds TB. Post-jejunoileal-bypass hepatic disease. Its similarity to alcoholic hepatic disease. Am J Clin Pathol. 1975;63(3):318-331.

73. Piepkorn MW, Mottet NK, Smuckler EA. Fatty metamorphosis of the liver associated with jejunoileal bypass. Report of five cases. Arch Pathol Lab Med. 1977;101(8):411-415.

74. D'Albuquerque LA, Gonzalez AM, Wahle RC, de Oliveira Souza E, Mancero JM, de Oliveira e Silva A. Liver transplantation for subacute hepatocellular failure due to massive steatohepatitis after bariatric surgery. Liver Transpl. 2008;14(6):881-885.

75. Andersen T, Gluud C, Franzmann MB, Christoffersen P. Hepatic effects of dietary weight loss in morbidly obese subjects. J Hepatol. 1991;12(2):224-229.

76. Burza MA, Romeo S, Kotronen A, et al. Long-term effect of bariatric surgery on liver enzymes in the Swedish Obese Subjects (SOS) study. PLoS One. 2013;8(3):e60495.

77. Mathurin P, Hollebecque A, Arnalsteen L, et al. Prospective study of the long-term effects of bariatric surgery on liver injury in patients without advanced disease. Gastroenterology. 2009;137(2): 532-540.
78. Stratopoulos C, Papakonstantinou A, Terzis I, et al. Changes in liver histology accompanying massive weight loss after gastroplasty for morbid obesity. Obes Surg. 2005;15(8):1154-1160.

79. Dixon JB, Bhathal PS, O'Brien PE. Weight loss and nonalcoholic fatty liver disease: falls in gamma-glutamyl transferase concentrations are associated with histologic improvement. Obes Surg. 2006;16(10):1278-1286.

80. Marceau P, Biron S, Hould FS, et al. Duodenal switch: long-term results. Obesity Surg. 2007;17(11):1421-1430

81. Kral JG, Thung SN, Biron S, et al. Effects of surgical treatment of the metabolic syndrome on liver fibrosis and cirrhosis. Surgery. 2004;135(1):48-58.

82. Keshishian A, Zahriya K, Willes EB. Duodenal switch has no detrimental effects on hepatic function and improves hepatic steatohepatitis after 6 months. Obes Surg. 2005;15(10):1418-1423.

83. Silverman EM, Sapala JA, Appelman HD. Regression of hepatic steatosis in morbidly obese persons after gastric bypass. Am J Clin Pathol. 1995;104(1):23-31.

84. Clark JM, Alkhuraishi AR, Solga SF, Alli P, Diehl AM, Magnuson TH Roux-en-Y gastric bypass improves liver histology in patients with non-alcoholic fatty liver disease. Obes Res. 2005;13(7):1180-1186.

85. Mattar SG, Velcu LM, Rabinovitz M, et al. Surgically-induced weight loss significantly improves nonalcoholic fatty liver disease and the metabolic syndrome. Ann Surg. 2005;242(4):610-617; discussion 618-620.

86. Mottin CC, Moretto M, Padoin AV, et al. Histological behavior of hepatic steatosis in morbidly obese patients after weight loss induced by bariatric surgery. Obes Surg. 2005;15(6):788-793.

87. Barker KB, Palekar NA, Bowers SP, Goldberg JE, Pulcini JP, Harrison SA. Non-alcoholic steatohepatitis: effect of Roux-en-Y gastric bypass surgery. Am J Gastroenterol. 2006;101(2):368-373.

88. Csendes A, Smok G, Burgos AM. Histological findings in the liver before and after gastric bypass. Obes Surg. 2006;16(5):607-611.

89. de Almeida SR, Rocha PR, Sanches MD, et al. Roux-en-Y gastric bypass improves the nonalcoholic steatohepatitis (NASH) of morbid obesity. Obes Surg. 2006;16(3):270-278.

90. Furuya CK Jr, de Oliveira CP, de Mello ES, et al. Effects of bariatric surgery on nonalcoholic fatty liver disease: preliminary findings after 2 years. J Gastroenterol Hepatol. 2007;22(4):510-514.

91. Liu X, Lazenby AJ, Clements RH, Jhala N, Abrams GA. Resolution of nonalcoholic steatohepatits after gastric bypass surgery. Obes Surg. 2007;17(4):486-492.

92. Weiner RA. Surgical treatment of non-alcoholic steatohepatitis and non-alcoholic fatty liver disease. Dig Dis. 2010;28(1):274-279.

93. Moretto M, Kupski C, da Silva VD, Padoin AV, Mottin CC. Effect of bariatric surgery on liver fibrosis. Obes Surg. 2012;22(7):1044-1049.

94. Klein S, Mittendorfer B, Eagon JC, et al. Gastric bypass surgery improves metabolic and hepatic abnormalities associated with nonalcoholic fatty liver disease. Gastroenterology. 2006;130(6): 1564-1572.

95. Tai CM, Huang CK, Hwang JC, et al. Improvement of nonalcoholic fatty liver disease after bariatric surgery in morbidly obese Chinese patients. Obes Surg. 2012;22(7):1016-1021.

96. Ratziu V, Charlotte F, Heurtier A, et al; LIDO Study Group. Sampling variability of liver biopsy in nonalcoholic fatty liver disease. Gastroenterology. 2005;128(7):1898-1906.

97. Rubino F. From bariatric to metabolic surgery: definition of a new discipline and implications for clinical practice. Curr Atheroscler Rep. 2013;15(12):369.

98. Miras AD, le Roux CW. Mechanisms underlying weight loss after bariatric surgery. Nat Rev Gastroenterol Hepatol. 2013;10(10): 575-584.

99. Buchwald H, Avidor Y, Braunwald E, et al. Bariatric surgery: a systematic review and meta-analysis. JAMA. 2004;292(14):1724-1737.

100. Garcia-Marirrodriga I, Amaya-Romero C, Ruiz-Diaz GP, et al. Evolution of lipid profiles after bariatric surgery. Obes Surg. 2012;22(4):609-616. 
101. Rao RS, Yanagisawa R, Kini S. Insulin resistance and bariatric surgery. Obes Rev. 2012;13(4):316-328.

102. Illán-Gómez F, Gonzálvez-Ortega M, Orea-Soler I, et al. Obesity and inflammation: change in adiponectin, C-reactive protein, tumour necrosis factor-alpha and interleukin-6 after bariatric surgery. Obes Surg. 2012;22(6):950-955.

103. Vilarrasa N, Vendrell J, Sánchez-Santos R, et al. Effect of weight loss induced by gastric bypass on proinflammatory interleukin-18, soluble tumour necrosis factor-alpha receptors, C-reactive protein and adiponectin in morbidly obese patients. Clin Endocrinol (Oxf). 2007;67(5):679-686.

104. Hosseinzadeh-Attar MJ, Golpaie A, Janani L, Derakhshanian H. Effect of weight reduction following bariatric surgery on serum visfatin and adiponectin levels in morbidly obese subjects. Obes Facts. 2013;6(2):193-202.

105. Sweeney TE, Morton JM. The human gut microbiome: a review of the effect of obesity and surgically induced weight loss. JAMA Surg. 2013;148(6):563-569.

106. Li JV, Ashrafian H, Bueter M, et al. Metabolic surgery profoundly influences gut microbial-host metabolic cross-talk. Gut. 2011;60(9):1214-1223.
107. Salehi M, Prigeon RL, D'Alessio DA. Gastric bypass surgery enhances glucagon-like peptide 1-stimulated postprandial insulin secretion in humans. Diabetes. 2011;60(9):2308-2314.

108. Rao RS, Kini S. GIP and bariatric surgery. Obes Surg. 2011;21(2):244-252.

109. Ben-Shlomo S, Zvibel I, Shnell M, et al. Glucagon-like peptide-1 reduces hepatic lipogenesis via activation of AMP-activated protein kinase. J Hepatol. 2011;54(6):1214-1223.

110. Al Massadi O, Lear PV, Müller TD, et al. Review of Novel Aspects of the Regulation of Ghrelin Secretion. Curr Drug Metab. May 5, 2014.

111. Manning S, Batterham RL. The role of gut hormone peptide YY in energy and glucose homeostasis: twelve years on. Annu Rev Physiol. 2014;76:585-608.

112. Burcelin R, Gourdy P, Dalle S. GLP-1-based strategies: a physiological analysis of differential mode of action. Physiology (Bethesda). 2014;29(2):108-121.

\section{Publish your work in this journal}

Hepatic Medicine: Evidence and Research is an international, peerreviewed, open access journal covering all aspects of adult and pediatric hepatology in the clinic and laboratory including the following topics: Pathology, pathophysiology of hepatic disease; Investigation and treatment of hepatic disease; Pharmacology of drugs used for

\section{Dovepress}

the treatment of hepatic disease. Issues of patient safety and quality of care will also be considered. The manuscript management system is completely online and includes a very quick and fair peer-review system, which is all easy to use. Visit http://www.dovepress.com/ testimonials.php to read real quotes from published authors. 